\title{
Evaluación de conocimientos, prácticas y prevención de la encefalitis equina venezolana en zonas rurales de Córdoba
}

\author{
Evaluation of knowledges, practices and prevention of \\ Venezuelan Equine Encephalitis in rural areas of Cordoba
}

\author{
Camilo Guzmán-Terán ${ }^{1}$ Alfonso Calderón-Range ${ }^{2}$ Hiltony Villa $^{3}$
}

Recibido para publicación: Marzo 022020 - Aprobado para publicación: Noviembre 252020

\begin{abstract}
RESUMEN
Introducción. La encefalitis equina venezolana (EEV) es una enfermedad zoonótica de origen viral, transmitida por mosquitos, principalmente de la especie Culex. Las enfermedades transmitidas por mosquitos representan una carga para la sociedad. Sin embargo, se pueden prevenir a través de conocimientos y buenas prácticas. Objetivo. Evaluar el nivel de conocimiento y participación de la comunidad en el control y prevención de EEV en algunas áreas rurales del departamento de Córdoba. Materiales y Métodos. Se realizó un estudio empírico analítico de corte transversal en 11 áreas rurales, con la aplicación de una encuesta de conocimientos, actitud y práctica para establecer una medición numérica. La fase de capacitación se hizo en las veredas La Cristalina en Puerto Escondido y Campo Bello en Tierralta (Córdoba). Resultados: Se entrevistó a un total de 95 hogares. La edad promedio de los encuestados fue 52 años. El 80.22\% fueron hombres. El nivel educativo alcanzado fue: primaria el $35.16 \%$, bachillerato el $29.67 \%$, técnica $5.49 \%$ y universitaria el $4.40 \%$. El $39.56 \%$ de la población evaluada consideró que la EEV es una enfermedad contagiosa, el $47.25 \%$ como una enfermedad mortal y el $5.49 \%$ como una molestia pasajera. Conclusión. La población no tiene claro el conocimiento sobre la EEV y el ciclo del vector, pese a esto la población encuestada tiene una buena actitud y disposición de cambio de estrategias para el control de las arbovirosis
\end{abstract}

Palabras clave: arbovirus, culex, dípteros, encefalitis, infecciones por alphavirus (DeCS).

\begin{abstract}
Introduction. Venezuelan equine encephalitis (VEE) is a zoonotic disease of viral origin, transmitted by mosquitoes, mainly of the Culex species. Mosquito-borne diseases represent a burden on society. However, they can be prevented through knowledge and good practices. Objective. To evaluate the level of knowledge and participation of the community in the control and prevention of VEE in some rural areas of the department of Córdoba. Materials and Methods. A cross-sectional analytical empirical study was carried out in 11 rural areas, with the application of a survey of knowledge, attitude and practice to establish a numerical measurement. The training phase was carried out in the villages of La Cristalina in Puerto Escondido and Campo Bello in Tierralta (Córdoba). Results. A total of 95 households were interviewed. The average age of the respondents was 52 years. $80.22 \%$ were men. The educational level reached was: primary $35.16 \%$, high school $29.67 \%$, technical $5.49 \%$ and university $4.40 \% .39 .56 \%$ of the population evaluated considered VEE to be a contagious disease, $47.25 \%$ as a fatal disease and $5.49 \%$ as a temporary annoyance. Conclusion. The population is not clear about the knowledge about VEE and the vector cycle, despite this the population studied has a good attitude and disposition to change strategies for the control of arboviruses.
\end{abstract}

Keywords: alphavirus infections, arboviruses, culex, diptera, encephalitis (DeCS).

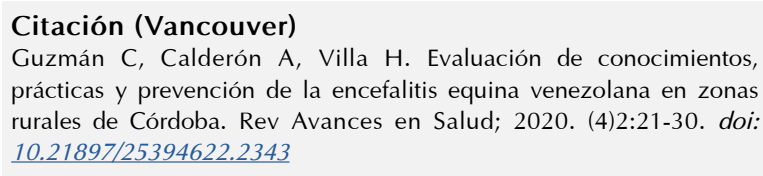

๑) 2020. Universidad de Cordoba. Este es un artículo de acceso abierto distribuido bajo los términos de la licencia Creative Commons Attribution License, que permite el uso ilimitado, distribución y reproducción en cualquier medio, siempre que el autor original y la fuente se acreditan.

\footnotetext{
${ }^{1}$ Ph.D Facultad de Ciencias de la Salud, Universidad de Córdoba. Correspondencia: cantonioguzman@correo.unicordoba.edu.co 2 Ph.D. Instituto de Investigaciones Biológicas del Trópico (IIBT). Colombia.

${ }_{3}^{3}$ MSc. Universidad de Córdoba. Colombia.
} 


\section{INTRODUCCIÓN}

El virus de la encefalitis equina venezolana (VEEV), es un miembro del género alphavirus de la familia Togaviridae; se transmiten principalmente por la picadura de mosquitos infectados (arbovirus) de las especies: Aedes, Anopheles, Culex, Psorophora, Wyeomya y Simulium; siendo las aves, roedores y caballos reservorios del virus. Su genoma es ARN, con envoltura compuesta por una bicapa lipídica derivada de la célula hospedadora y espículas, cada espícula está formada por 3 heterodímeros de las glicoproteínas E1 y E2, de la nucleocápside icosaédrica. Estos virus son endémicos en América Central y del Sur, Sin embargo, el virus se ha reportado en el sur de los Estados Unidos como La Florida y en la frontera con México. El VEEV puede afectar a todas las especies equinas, como caballos, burros y cebras; los equinos pueden morir repentinamente o mostrar trastornos progresivos del sistema nervioso central. Este virus es altamente infeccioso y especialmente el $100 \%$ de las personas expuestas desarrollan la enfermedad, aunque su tasa de mortalidad es relativamente baja; los síntomas varían desde fiebre, escalofríos, dolor de cabeza, malestar general, náuseas, vómitos, dolor abdominal, mialgias y dolor de garganta. Las personas con sistemas inmunes débiles como jóvenes y ancianos pueden enfermarse gravemente o morir a causa de las complicaciones del sistema nervioso central $(1,2)$.

En Colombia, el virus de la encefalitis equina venezolana (VEEV) se ha diagnosticado en los departamentos de la Costa Atlántica (Bolívar, Córdoba y Sucre) y otros departamentos como Antioquia, Cauca, Valle del Cauca, Tolima, Huila, Cundinamarca, Caquetá, Casanare, Arauca, Meta y Santanderes. Las epizoodemias del VEEV han ocurrido con un intervalo entre 15 y 20 años, especialmente en aquellos departamentos con altos niveles de precipitaciones estacionales, el
VEEV fue diagnosticado en Colombia, por primera vez en humanos en 1952 bajo condiciones naturales en el Espinal (Tolima), entre 1955 y 1959 se presentaron extensas epizootias en Colombia, para 1955 y 1962 se realizaron aislamientos repetidos del virus enzoótico en humanos con infección natural en San Vicente del Chucurí (Santander) en ausencia de epidemia. La epidemia del VEEV de mayor impacto, se inició en 1962 en la Guajira, de octubre a diciembre de ese año causando 3.000 casos humanos con 20 muertos en Colombia y en Venezuela, 6.762 casos con 43 muertes $(3,4)$.

En 1969, se realizó un estudio de prevalencia de la infección por el VEEV en humanos y équidos en diez departamentos colombianos, los resultados mostraron una prevalencia de anticuerpos contra el VEEV del $73 \%$ en los équidos y del $16 \%$ en humanos. La positividad en los équidos de la Costa Atlántica (Córdoba, Sucre, Atlántico, Magdalena y Cesar) estuvo entre el 68 al $93 \%$, en el Alto Magdalena (Tolima y Huila) entre el 56 al $75 \%$ y en el Valle del Cauca y el Valle del Patía del 37 al $40 \%$, los datos en humanos presentaron una distribución similar $(3,4)$.

En 1995, el brote del VEEV en Venezuela y Colombia fue el resultado de diferentes factores independientes como: vacunación insuficiente delosequinos,faltadevigilanciaepidemiológica sostenida, conocimiento limitado de la ecología de la encefalitis equina, $y$ un nivel de actividad viral más alto en las zonas donde la enfermedad ha estado presente. Para el 2016, el Instituto colombiano agropecuario (ICA) reportó tres casos de encefalitis equina en Córdoba (5), y recientemente, se reportó la circulación de este virus en murciélagos no hematófagos en dos zonas del departamento de Córdoba (6).

Colombia, tiene una política descentralizada y un compromiso para adelantar programas oficiales de prevención y control de las zoonosis, cumpliendo con estándares internacionales, 
notificando los focos que se registren en el territorio nacional a través de los sistemas de información oficial nacional e internacionales (7); ya que las Encefalitis Equinas Venezolana, del Este y del Oeste hacen parte de la lista de enfermedades definida por la Organización Mundial de Sanidad Animal (OIE) como enfermedades, infecciones e infestaciones comunes a varias especies donde se incluyen las enfermedades notificables desde el punto de vista económico y sanitario (8). Los equinos por ser amplificadores del VEEV, resultan ser un elemento fundamental para prevenir el riesgo en los humanos; por lo que la prevención de la enfermedad en los équidos es fundamental a través de la vacunación periódica (9).

El Instituto Colombiano Agropecuario (ICA) ejerce la vigilancia y control de la Encefalitis Equina en los animales susceptibles a través de un programa oficial de control de carácter nacional, y coordina acciones con las autoridades de salud pública para la prevención en la población humana (10). El objetivo de la presente investigación fue evaluar el nivel de conocimiento y participación en comunidades rurales en el control y prevención de EEV y capacitar a dos comunidades rurales en el control de algunas arbovirosis.

\section{MATERIALES Y MÉTODOS}

Tipo de estudio. Se realizó un estudio empírico analítico de corte transversal, usando recolección de datos para establecer medición numérica y análisis estadístico y así realizar interpretaciones y establecer patrones de comportamiento.

Área de estudio. La evaluación sobre conocimientos epidemiológicos se hizo en diferentes grupos poblacionales de áreas rurales del departamento de Córdoba y la fase de capacitación se hizo en dos comunidades rurales de las veredas La Cristalina en el municipio de Puerto Escondido y Campo Bello en Tierralta (Córdoba).

Tamaño de la muestra. Mediante un diseño no probabilístico, se aplicó una encuesta tipo Conocimiento, Actitudes y Prácticas (CAP) mediante visita domiciliaria casa a casa a 95 personas de ambos sexos, mayores de edad y residentes en áreas rurales de once municipios de Córdoba. La información que se compila sobre conocimientos, actitudes y prácticas de prevención es útil para evaluar el impacto de estrategias de educación, identificar determinantes de los conocimientos y prácticas o describir una situación de partida, para el diseño e implantación de estrategias comunitarias.

Fuentes y técnicas para la recolección de la información. Las fuentes de la información fueron establecidas así: Investigación bibliográfica: Se generó un listado sobre la legislación de políticas públicas sobre las encefalitis equinas y recolección de los datos epidemiológicos y demográficos de las poblaciones objeto de análisis, entre el 2014 y 2017; disponible en los portales de las entidades de control (ICA, INS). Investigación cualitativa: establecida desde la información recolectada desde las encuestas aplicadas y la información recolectada por entrevista directa con autoridades municipales y entes de salud.

Aspectos éticos. Previo a la entrega del formulario de la encuesta, se informó el objetivo del presente estudio y se invitó a participar a los entrevistados; en algunos casos por el nivel de educación se explicaron las preguntas y posibles respuestas del formulario, tratando de no inducir respuesta. Para facilitar la entrevista y diligenciamiento de la encuesta todas las variables se trataron de construir bajo un modelo categórico ( $\mathrm{Si}-\mathrm{No}$ ). 


\section{RESULTADOS}

Respuestas de la encuesta:

El $80.22 \%$ de las personas que respondieron la encuesta fueron hombres y el $19.78 \%$ mujeres; el promedio de edad fue de 52 años con un valor mínimo de 17 y un máximo de 62 años.

La ocupación de la población evaluada fue conformada por estudiantes el $13.19 \%$, empleados el $47,25 \%$, amas de casa el 31,87\% y trabajadores independientes el $7.69 \%$. El nivel educativo alcanzado fue: primaria el $35.16 \%$, bachillerato el $29.67 \%$, técnica $5.49 \%$ y universitaria el $4.40 \%$. En la figura

1. Se muestran los resultados del nivel de conocimientos de los ciudadanos encuestados en zonas rurales del departamento de Córdoba con respecto al virus de la encefalitis equina venezolana.

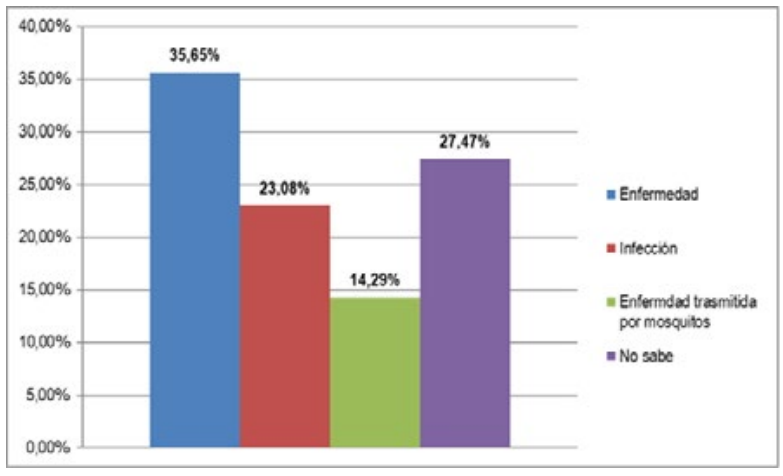

Gráfica 1. Nivel de conocimientos frente al VEEV en pobladores de algunas zonas rurales del departamento de Córdoba. Montería Córdoba 2020.

La población evaluada, consideró que el grado de afectación del VEEV fue: niños con el $43.96 \%$, jóvenes el $17.58 \%$, ancianos el $10.99 \%$ y el $21.98 \%$ reconoció que el VEEV puede afectar a cualquier grupo poblacional descrito anteriormente.

Sobrelos síntomas del VEEV, el 43.96\% relacionó dolores fuertes de cabeza con encefalitis, el $24.18 \%$ con fiebre, el $16.48 \%$ con caídas de presión o debilidad, el $10.99 \%$ con una gripa intensa y el $4.40 \%$ con dolores articulares y musculares. En la figura 2 encontramos el nivel de conocimientos sobre el agente causal de la encefalitis equina venezolana en pobladores de algunas zonas rurales del departamento de Córdoba.

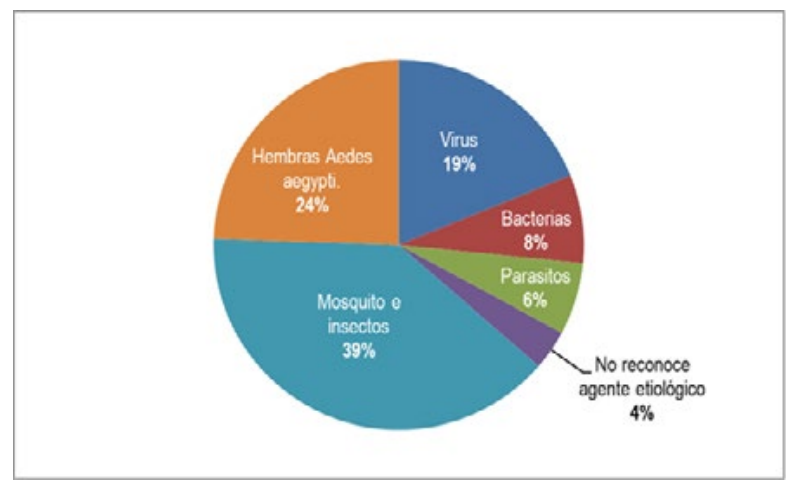

Gráfica 2. Conocimientos del agente causal del VEEV en pobladores encuestados de algunas zonas rurales del departamento de Córdoba.

Dentro de las acciones para prevenir la aparición del VEEV y otras arbovirosis, el $35.16 \%$ de la población evaluada consideró necesario el lavado constante de las albercas, el $12.09 \%$ respondió que no acumula agua en recipientes abiertos, el 9.89\% manifestó recoger materiales inservibles que pueden acumular agua, el $27.47 \%$ el uso de desinfectantes como el cloro y el $15.38 \%$ respondió que no toma ninguna medida profiláctica contra el VEEV. Igualmente, la población en estudio reconoció la frecuencia de lavado y desinfección de tanques y albercas fue: semanal $32.97 \%$, quincenal $27.47 \%$, mensual $21.98 \%$, semestral $17.58 \%$.

El $65.93 \%$ de la población evaluada consideró que la mejor forma de prevenir la picadura de mosquitos cuando duermen es el uso de toldillos y el $34.07 \%$ la fumigación. Dentro de los factores de riesgo en las viviendas para la presencia del mosquito, el 58.24\% consideró a los estanques de agua abiertos, el $28.57 \%$ a un mal lavado o ausencia de lavado, el $8.79 \%$ no consideró ningún factor de riesgo y el $4.40 \%$ nunca ha fumigado su vivienda. El método de almacenamiento del agua en las casas fue: $58.24 \%$ canecas plásticas con tapa, el $48.35 \%$ albercas y el $16.48 \%$ botellas plásticas. En la figura 3 se muestra la actitud de la población encuestada en caso probable de infectarse con 
el VEEV.

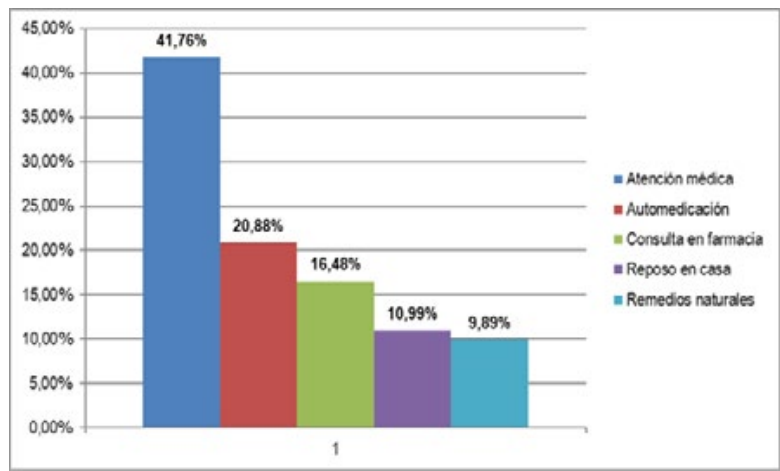

Gráfica 3. Actitud de la población evaluada en caso de infectarse con el VEEV.

El $18.68 \%$ consideró que la asistencia a charlas comunitarias no es importante, el $37.36 \%$ ha participado en actividades de limpieza y remoción de inservibles, el $43.96 \%$ abre puertas y ventanas cuando realizan fumigaciones.

El $39.56 \%$ de la población evaluada consideró que el VEEV es una enfermedad contagiosa, el $47.25 \%$ como una enfermedad mortal y el $5.49 \%$ como una molestia pasajera. Con relación a una capacitación futura para conocer sobre el VEEV, el $27.47 \%$ consideró aspectos relacionados con la prevención, el $35.16 \%$ sobre la trasmisión y el $20.88 \%$ sobre la sintomatología de la enfermedad.

Actividades de transferencia. Estos conceptos evaluados mediante encuestas realizadas por los estudiantes del Semillero del Instituto de Investigaciones Biológicas del Trópico (IIBT) en diferentes localidades de Córdoba, permitió implementar una fase de capacitación pedagógica en diferentes tópicos de prevención y control en zoonosis en habitantes de zonas rurales de los municipios de Puerto Escondido y Tierralta (Córdoba), por los casos presentados del síndrome febril indiferenciado.

La implementación de la fase pedagógica se hizo a través de charlas radiales de una hora de duración, con un relato oral breve a los oyentes que incluyó la presentación de los eventos epidemiológicos zoonóticos ocurridos en los municipios de Puerto Escondido y Tierralta, definición e importancia de las zoonosis, formas de prevención y control, interacción con entes territoriales, respuesta a los comentarios de los oyentes, finalmente una síntesis y conclusiones en colaboración con el director del programa Cuadernos del Campo de la emisora cultural de la Universidad de Córdoba.

Capacitación a estudiantes. Para esta capacitación, se seleccionaron los estudiantes de 4 y 5 grado de las Instituciones Educativas La Cristalina en Puerto Escondido y Campo Bello en Tierralta, con una asistencia de 29 estudiantes; en esta capacitación se contó con la colaboración de los biólogos que participaron en el proyecto "Murciélagos como hospederos de virus zoonóticos en dos zonas del Caribe colombiano (Fase 1)" y la capacitación fue dirigida a conservar y comprender conceptos básicos de la biología y la biodiversidad, conocer los servicios ambientales de los murciélagos como controladores de poblaciones de insectos, propagadores de semillas y polinización; estimular la observación crítica en los procesos ecológicos y sociales que ocurren con la presencia humana.

Capacitación en habitantes y productores rurales. El objetivo de estas charlas fue socializar estrategias de prevención y control, mediante un diálogo entre un equipo de expertos (investigadores y funcionarios de la Secretaria de Desarrollo de la Salud del Departamento de Córdoba) y las comunidades rurales para disminuir el riesgo de la encefalitis equina venezolana. Durante la capacitación, se elaboró y se priorizó con la comunidad un esquema de prevención y control de las encefalitis equinas.

\section{DISCUSIÓN}

Los brotes de encefalitis equina no son muy frecuentes en el área de estudio, Sin embargo, posibles brotes podrían confundirseconDengue, Chikungunya, Zika, Malaria, Mayaro entre otras enfermedades febriles tropicales. A pesar de eso no existen estudios de conocimiento, actitud, práctica y control de la encefalitis equina venezolana en la región donde se llevóa 
cabo el estudio. Los hallazgos de la presente investigación mostraron que existe una buena actitud y prácticas con respecto al control del VEEV en la población local. Aunque el nivel de conocimiento fue muy bajo en nuestro estudio, sólo el 35.65\% de los encuestados escucharon hablar sobre el VEEV. Un hallazgo casi similar para el caso de la encefalitis japonesa (54\%) reportado por un estudio realizado en el distrito de Rupandehi en de Nepal (11). Otros estudios informaron un porcentaje menor (12-13). Un estudio CAP hecho en Malwa (India) sobre la enfermedad transmitida por mosquitos entre los estudiantes reveló que sólo el $6.5 \%$ sabía que la encefalitis japonesa es transmitida por mosquitos. Con respecto al nivel educativo bajo en los encuestados, es importante tenerlo en cuenta para realizar actividades educativas comunitarias utilizando un lenguaje sencillo alejado del lenguaje técnico pero que permita el conocimiento de aspectos ecológicos, biológicos y clínicos relacionados con la enfermedad.

Los síntomas típicos de la encefalitis equina venezolana como fiebre, dolor de cabeza y convulsiones, fueron reportados como los más frecuentes. El $61.54 \%$ de los encuestados reconoció que el VEEV es transmitido por mosquitos, sin embargo, el conocimiento sobre el sitio de reproducción del mosquito fue bajo (35.16\%) en este estudio. En contraste, Shrestha et al., 2005 en un estudio sobre la encefalitis japonesa observaron un mayor nivel $(50 \%)$ de conciencia sobre el lugar de reproducción (14); mientras el estudio de Karnataka (India) tuvo observación similar al del presente estudio (15).

La conciencia sobre los síntomas del VEEV fue baja en nuestro estudio, el $100 \%$ de las personas no pudieron decir cuáles eran los síntomas clásicos de la encefalitis equina venezolana en contraste con un estudio sobre encefalitis japonesa donde fue del $61.3 \%$ (16). Con respecto a los sitios de cría de los mosquitos el $58.24 \%$ de los encuestados consideró a los estanques de agua abiertos. La actitud fue pobre con respecto al control de los sitios de reproducción y las medidas tomadas para evitar potenciales crías de mosquitos Un estudio similar en Puducherry (India) coincide con nuestros resultados, donde el $70 \%$ de las personas almacenaron agua en los hogares (17). Este tipo de práctica comunitaria favorece la cría de mosquitos. (18).

Existen varios aspectos que contribuyen a la probable presencia del VEEV y de otras enfermedades tropicales transmitida por vectores artrópodos como el Zika, Dengue, Chikungunya, asociados a la conducta humana entre los que se encuentran el almacenamiento de agua en forma inadecuada, la cultura generalizada de conservar inservibles $o$ recipientes a cielo abierto y la falta de compromiso de la comunidad en la prevención de la enfermedad. La mejor forma de prevenir el VEEV igual que otras enfermedades tropicales es eliminar todos los criaderos de mosquitos. Como no existen vacunas que prevengan el VEEV en humanos y carencia de tratamiento farmacológico la medida más importante de prevención es la eliminación de todos los criaderos de mosquitos, es decir, de todos los recipientes que contienen agua tanto en el interior de las casas como en sus alrededores. Muchos de los recipientes donde el mosquito se cría no son de utilidad (latas, botellas, neumáticos, trozos de plástico y lona, bidones cortados). Estos recipientes deben ser eliminados. Si los recipientes no pueden eliminarse porque se usan permanentemente debe evitarse que acumulen agua, haciendo lavados periódicos con desinfectantes a base de hipoclorito.

Respecto a la actitud de la población evaluada de acudir a la atención médica fue baja. Dado que al presentar síntomas similares a la influenza se automedican esta práctica está muy arraigada en la comunidad. El cuadro clínico inicial del VEEV es indistinguible de la influenza, dengue y otras enfermedades febriles tropicales. Esta actitud complica la situación. Además, la práctica de automedicarse está influenciada por el mal servicio prestado por las entidades territoriales de salud, quienes sólo atienden pacientes bajo situaciones 
complicadas y de urgencia.

En la atención de casos primarios de VEEV en los animales no hay tratamiento específico y las medidas de prevención y control van encaminadas a la implementación masiva de la aplicación de vacunas de virus atenuados de la Encefalomielitis Equina Venezolana Cepa TC83, en zonas endémicas o zonas por debajo de los 1200 metros de altitud sobre el nivel del mar. Esta vacuna induce la producción de anticuerpos post-vacunales por 2 años. Las medidas de prevención incluyen control de las densidades de mosquitos mediante la educación a la población, destrucción de reservorios de mosquitos mediante el empleo de medidas culturales como la recolección de inservibles, control de sumideros, protección de la fauna silvestre controladora de insectos y uso de productos químicos o de biológicos (19-20). El Instituto Colombiano Agropecuario (ICA), autoridad sanitaria en Colombia, ha establecido dentro de los requisitos de movilización el registro de vacunación contra el VEEV (21).

\section{CONCLUSIONES}

Los conocimientos sobre encefalitis equina venezolana son deficientes, las actitudes son buenas, aunque no óptimas, pero las prácticas de control son muy pobres, por la poca participación de la comunidad. Se requiere incentivar acciones que motiven a las personas a mejorar sus conocimientos para implementar las prácticas de prevención como el control de dípteros. En las enfermedades por arbovirus es muy importante la participación comunitaria por el apoyo a las actividades colectivas. Es necesario que las comunidades se empoderen de su papel para asumir la prevención y el control de las Encefalitis Equina Venezolana, Dengue, Zika, Chikungunya, Mayaro entre otras como parte de sus actividades.

\section{CONFLICTO DE INTERÉS}

Los autores declaran no tener conflicto de interés.

\section{REFERENCIAS}

1. Sharma A, Knollmann-Ritschel B. Current understanding of the molecular basis of Venezuelan Equine Encephalitis Virus pathogenesis and vaccine development. Viruses. 2019;11(2):164. doi: 10.3390/ v11020164.

2. Kumar B, Manuja A, Gulati BR, Virmani $\mathrm{N}$, Tripathi $\mathrm{BN}$. Zoonotic viral diseases of equines and their impact on human and animal health. Open Virol J. 2018; 12:8098. doi: 10.2174 / 1874357901812010080

3. Mesa FA, Cárdenas ZA, Villamil JLC. Las encefalitis equinas en la salud pública. 2005. Departamento de Salud Animal, Facultad de Medicina Veterinaria y de Zootecnia, Universidad Nacional de Colombia

4. Aguilar PV, Estrada-Franco JG, NavarroLópez R, Ferro C, Haddow AD, Weaver SC. Endemic Venezuelan equine encephalitis in the Americas: hidden under the dengue umbrella. Future Virol. 2011; 6(6): pp. 721740. doi:10.2217/FVL.11.5

5. Instituto Colombiano Agropecuario. Dirección Técnica de Vigilancia Epidemiológica. Subgerencia de Protección Animal. Sanidad Animal-2016. Disponible en: https://www.ica.gov.co/ getattachment/6d2f08b5-da5d-49a2ad3c-ef3ccfe06df7/Boletin-2016-SanidadAnimal.aspx

6. Guzmán C, Calderón A, Martinez C, Oviedo M, Mattar S. Eco-epidemiology of the Venezuelan equine encephalitis virus in bats of Córdoba and Sucre, Colombia. Acta Trop. 2019;191:178-184. doi:10.1016/j. actatropica.2018.12.016

7. Agudelo-Suárez Ángela N. VillamilJiménez Luis C. Políticas de zoonosis en Colombia: del Código Sanitario a la salud ambiental. Rev. Salud Pública. 2018; 20(1): 34-44.

8. Organización Mundial de Sanidad Animal. Información sobre las enfermedades de los animales acuáticos y terrestres. Disponible en: https://www.oie.int/es/sanidad-animal- 
en-el-mundo/enfermedades-de-losanimales/

9. Instituto Nacional de Salud. Vigilancia y análisis del riesgo en salud pública protocolo de vigilancia en salud publica encefalitis equinas, 2016. Disponible en: http://www.saludpereira.gov.co/medios/ Encefalitis_Equinas_PROTOCOLO2016. pdf

10. Instituto Colombiano Agropecuario. Resolución No. 1026 de 1999. Por la cual se establecen medidas sanitarias para la prevención y control de la Encefalitis Equina Venezolana. Disponible en: https:// www.ica.gov.co/normatividad/normasica/resoluciones-oficinas-nacionales/ resoluciones-derogadas/resol-1026de-1999.aspx

11. Khanal VK, Pyakurel $P$, Uprety $S$, Rayamajhi RB, Gupta PP, Ghimire A, et al. Mosquito borne diseases related knowledge, practice and behaviour of people in rural and peri-urban areas of Eastern Nepal. Health Renaiss. 2015;12(2): 92-8.

12. Dhakal S, Stephen C, Ale A, Joshi DD. Knowledge and practices of pig farmers regarding Japanese encephalitis in Kathmandu, Nepal. Zoonoses Public Health. 2012;59(8): 568-574.

13. Pandit N, Patel Y, Bhavsar B. Awareness and practice about preventive method against mosquito bite in Gujarat. Health Line. 2010;22(1):16-20.

14. Shrestha M, Gupta R, Gautam I. Epidemioentomological study of Japanese encephalitis in Bhaktapur District, Nepal. J Inst Sci Technol. 2015;20(1):112-118.
15. Mackenzie JS. Emerging zoonotic encephalitis viruses: Lessons from Southeast Asia and Oceania. J Neurovirol. 2005;11(5):434-440.

16. Yadav S, Ahmad S. An assessment of community participation in control and prevention of Japanese Encephalitis in rural Uttar Pradesh. Int J Med Sci Public Health. 2017; 6(11): 1614-1619.

17. Boratne AV, Jayanthi V, Datta SS, Singh Z, Senthilvel V, Joice YS. Predictors of knowledge of selected mosquito-borne diseases among adults of selected periurban areas of Puducherry. J Vector Borne Dis. 2010;47(4): 249-256.

18. DhadukKM, Gandha KM, Vadera BN, Mehta JP, Parmar DV, Yadav SB. A community level KAP study on mosquito control in Jamnagar district. Natl J Community Med. 2013;4(2):321-328.

19. Vargas DS, Jaime CJ, Vera VJ. Aspectos generales del virus de la encefalitis equina venezolana (VEEV). Orinoquia. 2009;13(1):59-67.

20. Salinas-López MA, Soto-Rojas VE, Ocampo CB. Costos de un programa de control del vector Aedes aegypti en municipios de Colombia: el caso de Girón y Guadalajara de Buga, 2016. Cad Saúde Pública. 2018;34(12): e00044518.

21. Instituto Colombiano Agropecuario. Encefalitis equina. Disponible en: https:// https://www.ica.gov.co/getdoc/0408af84$7210-4 d 0 d-b a 47-74$ a a 903 cbf $48 /$ encefalitis-equina-(1).aspx 